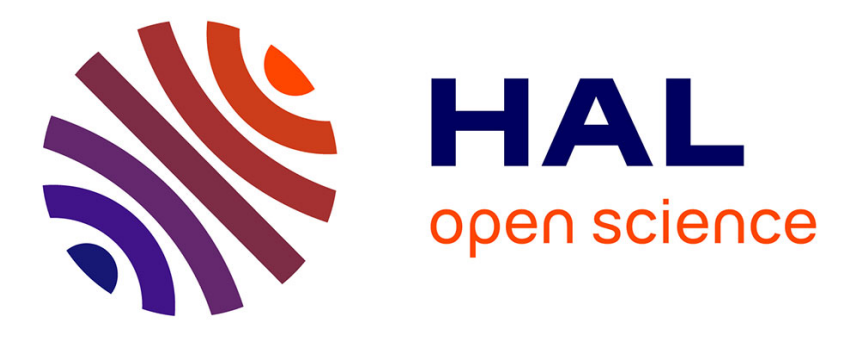

\title{
Kron Simulation of Field-to-line Coupling Using a Meshed and a Modified Taylor Cell
}

Sjoerd Op 'T Land, Richard Perdriau, Mohamed Ramdani, Olivier Maurice, M'Hamed Drissi

\section{- To cite this version:}

Sjoerd Op 'T Land, Richard Perdriau, Mohamed Ramdani, Olivier Maurice, M'Hamed Drissi. Kron Simulation of Field-to-line Coupling Using a Meshed and a Modified Taylor Cell. Electromagnetic Compatibility of Integrated Circuits (EMC Compo 2013), The 9th International Workshop on, Dec 2013, Nara, Japan. pp.1-6. hal-00923660

\section{HAL Id: hal-00923660 https://hal.science/hal-00923660}

Submitted on 3 Jan 2014

HAL is a multi-disciplinary open access archive for the deposit and dissemination of scientific research documents, whether they are published or not. The documents may come from teaching and research institutions in France or abroad, or from public or private research centers.
L'archive ouverte pluridisciplinaire HAL, est destinée au dépôt et à la diffusion de documents scientifiques de niveau recherche, publiés ou non, émanant des établissements d'enseignement et de recherche français ou étrangers, des laboratoires publics ou privés. 


\title{
Kron Simulation of Field-to-line Coupling Using a Meshed and a Modified Taylor Cell
}

\author{
Sjoerd Op 't Land*, Richard Perdriau*, Mohamed Ramdani*, Olivier Maurice ${ }^{\dagger}, \mathrm{M}^{*}$ 'hamed Drissi ${ }^{\ddagger}$ \\ ${ }^{*}$ Department of Electronics, Ecole Superieure d'Electronique de Ouest (ESEO), Angers, France \\ Email: \{sjoerd.optland, richard.perdriau, mohamed.ramdani\}@eseo.fr \\ †Groupe d'Études et de Recherches Appliquées à la Compatibilité Électromagnétique (GERAC), Trappes, France \\ Email: olivier.maurice@gerac.fr \\ *Université européenne de Bretagne IETR, Rennes, France \\ Email: mhamed.drissi@insa-rennes.fr
}

\begin{abstract}
Printed Circuit Board (PCB) traces play a role in the immunity of electronic products. Contrary to Integrated Circuits (ICs), the layout of PCB traces can be changed rather late in a product's design. Therefore, it is interesting to equip the PCB designer with simple tools that predict the immunity of his PCB traces.

In this article, we compare two simulations of field-to-long line coupling based on Taylor's model. Firstly, the line is meshed into electrically short Taylor cells and numerically simulated using Kron's method. Secondly, we use one modified Taylor cell, which does not need meshing and is a closed-form, analytical result.

The two simulations turn out to be equally precise on a straight microstrip line, the meshed simulation being more flexible, the simulation using a modified Taylor cell being faster.

Index Terms-PCB, EMC, field-to-line coupling, immunity, microstrip, Kron, frequency-adaptive meshing, modified Taylor
\end{abstract}

\section{INTRODUCTION}

Electromagnetic compatibility (EMC) problems can often be understood as a three-element chain: agressor-coupling path-victim [1]. In the case of unshielded, wireless electronics, the dominant coupling path can consist in the PCB traces. Therefore, the routing of PCB traces may be decisive for product compliance.

In contrast to integrated circuits (ICs), PCB layout may be changed rather easily and in a late design stage. Field-to-line coupling models could help the PCB designer to predict and explain product immunity. The prediction helps the designer to detect problems before fabricating the first prototype, the explanation helps the designer to do something about the detected problems. As electromagnetic coupling is rarely easy to explain, even by humans, we do not believe in automatic explanation of product immunity. Very fast automatic prediction, however, would allow the designer to freely play around with his design and develop intuition for the coupling mechanisms. Note that this prediction need not be very precise, as long as it faithfully reveals the influence of the designable parameters. Therefore, the focus of this article will be on fast, numerical prediction of field-to-trace coupling.

We will now define a rather simple case study to evaluate the methods. However, we keep more realistic PCB traces in mind when concluding on their performance.
We choose a microstrip, i.e. a trace above a ground plane, because it is still widely used. Moreover, with respect to coplanar waveguides (CPWs) and striplines, it is good antenna and therefore prone to create immunity problems.

Operational and harmonic frequencies of electronics keep rising, so the wavelengths keep falling. For example, the Wireless Home Digital Interface (WHDI) uses a $5 \mathrm{GHz}$ carrier, or a $6 \mathrm{~cm}$ wavelength in free space. Back-up radars may use ultra-wideband signals up to $24 \mathrm{GHz}$, or down to $1.25 \mathrm{~cm}$. PCBs still have sizes in that order of magnitude, so we may expect long-line effects. Therefore, we choose to illuminate a $5 \mathrm{~cm}$ trace with a frequency up to $20 \mathrm{GHz}$.

In practice, traces are never characteristically terminated, because the terminating ICs and passives have frequency dependent impedances. Neither are real-world traces uniform, because of width changes and unmitered bends. However, we believe that there is already sufficient microwave theory to incorporate these non-idealities in simulation. Here, we would like to focus on modelling of field-to-trace coupling. Therefore, we allowed ourselves to study a uniform $50 \Omega$ trace that is characteristically terminated. According to typical technology, the $\varepsilon_{r}=4.6$ substrate is $362 \mu \mathrm{m}$ thick.

Finally, the most constraining simplification is that of grazing incidence (cf. Figure 1). The vertically polarised plane wave is not refracted by the air-substrate interface and the incident wave is simply doubled by the ground plane. The field strength in the dielectric substrate thus amounts to:

$$
\begin{aligned}
H & =2 H^{i} \\
E & =2 E^{i} / \varepsilon_{r} .
\end{aligned}
$$

For low frequencies, grazing incidence constitutes the worst case [2]. Also, it models Gigahertz Transverse ElectroMagnetic cell (GTEM-cell) measurements, which integrate a PCB in the waveguide wall. Otherwise, this is a serious limitation.

For the numerical calculation, the field generated by a standard GTEM cell will be entered: $1 \mathrm{~V}$ at a $50 \Omega$ septum, separated by $42 \mathrm{~mm}$ from the PCB. Hence, the terminal voltage in $\mathrm{dBV}$ is numerically equal to the $S_{21}$ coefficient that would be measured between GTEM input and trace terminal. 


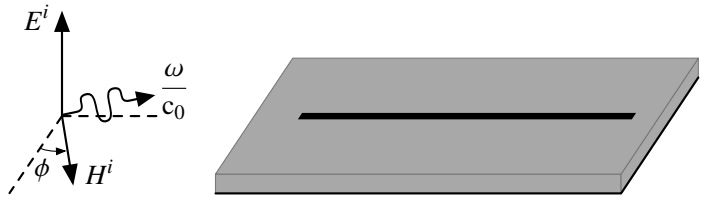

Figure 1. Grazing incidence: the incident far-field wave vector $k^{i}=\omega / \mathrm{c}_{0}$ is tangential to the substrate.

In section II, we will briefly review existing field-to-trace coupling models and pick Taylor's model to continue with. We will implement this model using frequency-adaptive meshing and Kron's method simulation in section III. Alternatively, we will explain and apply the modified Taylor's cell in section IV. Both simulations will be compared, conclusions drawn and suggestions for future research given in section VI.

\section{StATE OF THE ART}

Field-to-line coupling is complicated, like almost any reallife electromagnetic problem. Let us therefore start by reviewing four common simplifications.

If wavelengths are great with respect to the studied geometry, the quasi-static approximation may be used. The field then still changes with time, but propagates instantaneously everywhere. In that case, the illumination field is uniform. This approximation yields low-frequency asymptotes, useful for checking our model. However, as we specifically chose an electrically long line, we will not use this approximation.

In reality, everything interacts bilaterally. Indeed, the field emitted by an aggressor will couple to a guided wave in the victim line. However, this guided wave will also affect the aggressor. This, in turn, will affect the victim again, and so on. To predict what will happen, knowledge about the aggressor is needed. Because we suspect that there be only weak coupling and would like to avoid incorporating knowledge about the aggressor, we will only consider the unilateral interaction from field to line.

As long as the cross section of the microstrip transmission line remains small with respect to the wavelength, there is only one dominant mode: the differential Transversal ElectroMagnetic (TEM) mode. Because this assumption only gradually breaks down at several $\mathrm{GHz}$ for modern electronics [3], we choose to adopt this approximation. This allows us to use transmission line theory to describe the trace.

Finally, we will suppose traces to be lossless. Consequently, immunity predictions will be pessimistic with respect to reality, but only slightly [3].

There are three equivalent, weakly coupled, transmission linebased field-to-line coupling models [4]: that of Taylor et al. [5], of Agrawal et al. [6] and of Rachidi [7]. They all model the coupling of an illuminating field by means of current and/or voltage sources, distributed along the line. Agrawal and Rachidi also need sources at both terminals.

All of these models basically represent a uniform transmission line. Consequently, when modeling a piecewise non-

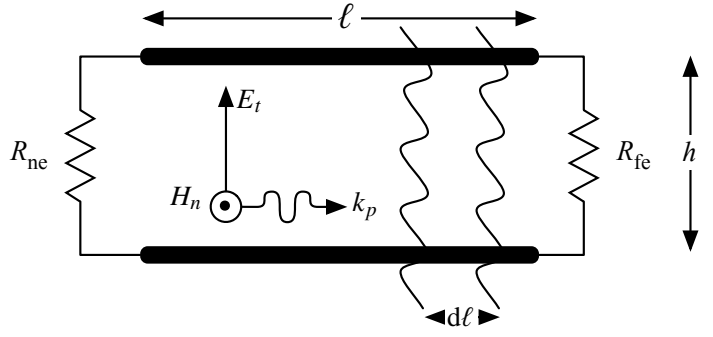

(a) Line geometry: the indices $n, t$ and $p$ indicate vector components normal to the plane of the wires, transversal to the line and parallel with the wires, respectively. $R_{\mathrm{ne}}$ and $R_{\mathrm{fe}}$ are the near-end and far-end resistive terminations.

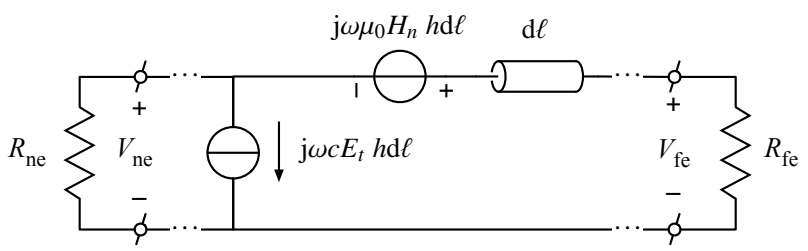

(b) Taylor cell: a current source models the electrostatic force (capacitive coupling) and a voltage source the electromotive force (emf or inductive coupling) in each line segment $\Delta \ell . c$ denotes the per-unit-length p.u.1.) capacity of the line.

Figure 2. Taylor's field to line coupling model for a bifilar transmission line.

uniform line using Agrawal or Rachidi, sources appear at the transitions. Using Taylor's model, on the other hand, only the distributed sources along the line change value, generally. That way, it is even possible to model continuously non-uniform lines. In view of the long-term goal to model non-uniform lines, we prefer the simplicity of Taylor's model and will continue with that model only.

The specialisation of Taylor's model for a two-wire transmission line in vacuum is shown in Figure 2. In each transmission line slice $\mathrm{d} \ell$, the magnetic field normal to the plane of the wires $H_{n}$ induces a voltage and the electric field in the plane and transversal to the wires $E_{t}$ induces a current.

\section{Meshed Taylor Simulation Using Kron}

The most obvious application of Taylor's model for a nonuniform incident field, is to mesh (segment) the line in short enough cells, in order for the field to become approximately uniform to each cell. The passive transmission line itself must also be modeled, for example as an rglc telegrapher's cell. As we are considering a lossless line, we omit the dissipative elements $r$ and $g$. The resulting model of a line for the case of three cells is depicted in Figure 3.

With increasing frequency, wavelength decreases and generally, the field becomes less uniform along the line. Therefore, a large number of cells may be needed to accurately model the line. In the perspective of a simple tool, we avoid manually entering this multitude of cells, because it is error-prone and time-consuming. We chose to analyse this problem in terms of Gabriel Kron's formalism [8], because of its promise to handle complex electromagnetic systems [9].

We will now first describe the basic approach of the problem in Kron's formalism. Then, we will describe the practical 


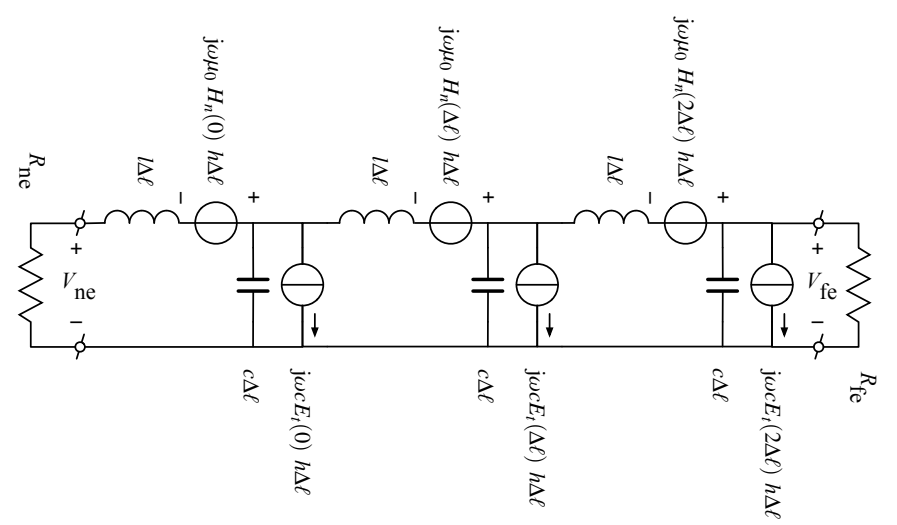

Figure 3. Lossless transmission line meshed in three cells $\left(\Delta \ell=\frac{1}{3} \ell\right)$. The passive transmission line is modeled with $l$ and $c$ being the per-unit-length inductance and capacity, respectively.

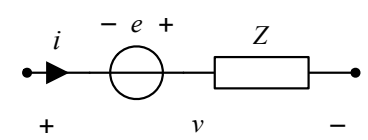

Figure 4. Simplified Kirchhoff branch. The difference of potential $v$ across the branch and the current $i$ through the branch are defined such, that when $i v$ is positive, net power is dissipated in the branch (passive sign convention).

implementation, including performance optimisation.

\section{A. Basic Approach}

Generally, solving a problem in Kron's formalism consists of eight steps: stating the problem, drawing the associated graph, define the topological base, entering the sources, transforming, solving in mesh space, deducing the differences of potentials and deducing other required quantities [10].

Let us draw the graph corresponding to the problem of Figure 3. In this graph, we identify meshes (or loops) and nodes (or junctions). Meshes consist of branches (or vertices) that each connect two nodes. We will here use simplified Kirchhoff branches, which generally consist of an impedance $Z$ and a voltage source $e$ as defined in Figure 4. The resulting graph is depicted in Figure 5.

Let $i, v$ and $e$ be column vectors in the branch space, that is: containing currents and voltages for every branch. The (arbitrary) branch numbers of Figure 5 define which vector component represents which voltage and current: we just defined the topological base. Ohm's and Kirchoff's laws then hold as in $v+e=Z i$. In our case, the impedance matrix

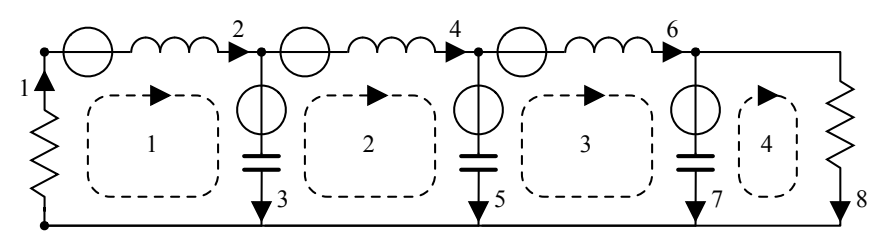

Figure 5. Graph representation of a three-cell transmission line model. Please verify that there are 4 meshes (dashed loops, numbered), 8 branches (with arrows, numbered) and 5 nodes (dots, not numbered).
$Z$ only has entries on its main diagonal:

$$
\begin{aligned}
\operatorname{diag}(Z)=\left[R_{\mathrm{ne}}, \mathrm{j} \omega l \Delta \ell, \frac{1}{\mathrm{j} \omega c \Delta \ell},\right. & \\
& \left.\mathrm{j} \omega l \Delta \ell, \frac{1}{\mathrm{j} \omega c \Delta \ell}, \mathrm{j} \omega l \Delta \ell, \frac{1}{\mathrm{j} \omega c \Delta \ell}, R_{\mathrm{fe}}\right] .
\end{aligned}
$$

To incorporate the current sources in the simplified Kirchhoff branch, we need to use their Thévenin equivalents $E_{t} h$. The source vector $e$ stemming from the illumination electromagnetic field thus becomes:

$$
e=\left[\begin{array}{ll}
0 & 0 \\
H_{n}(0) & 0 \\
0 & E_{t}(0) \\
H_{n}(\Delta \ell) & 0 \\
0 & E_{t}(\Delta \ell) \\
H_{n}(2 \Delta \ell) & 0 \\
0 & E_{t}(2 \Delta \ell) \\
0 & 0
\end{array}\right]\left[\begin{array}{c}
\mathrm{j} \omega \mu_{0} h \Delta \ell \\
h
\end{array}\right]
$$

To solve for the mesh currents, we need to transform our equations to another topological base: that of the mesh space. At the same time, we will connect the branches together. This is done by means of the connectivity matrix $L$, which links the branches (rows) with the meshes (columns). In our example,

$$
L=\left[\begin{array}{rrrr}
1 & 0 & 0 & 0 \\
1 & 0 & 0 & 0 \\
1 & -1 & 0 & 0 \\
0 & 1 & 0 & 0 \\
0 & 1 & -1 & 0 \\
0 & 0 & 1 & 0 \\
0 & 0 & 1 & -1 \\
0 & 0 & 0 & 1
\end{array}\right]
$$

Note that a minus signs signifies a branch going against the mesh direction. We will denote tensors in mesh space with a hat, e.g.:

$$
\hat{e}=L^{-1} e \quad i=L \hat{\imath} \quad \hat{v}=L^{-1} v \equiv \mathbf{0},
$$

where the last vector (voltage around every mesh) is zero by Kirchhoff's mesh rule. The inverse of $L$ can be found by its transpose, because $L$ always is a Hadamard matrix. We can transform Kirchoff's laws to mesh space as follows:

$$
\begin{aligned}
L^{-1} v+L^{-1} e & =L^{-1} Z i=L^{-1} Z L \hat{\imath} \\
\hat{e} & =\hat{Z} \hat{\imath} .
\end{aligned}
$$

Notice that by transforming to the lower-dimensional mesh space, we connected the branches together.

To solve the system, we use the pseudoinverse $\left(\right.$ denoted $\left.^{+}\right)$:

$$
\hat{\imath}=\hat{Z}^{+} \hat{e},
$$

because only the sources $e$ are given.

We are interested in the near-end and far-end voltages, which can now be found by means of the terminal impedances:

$$
\begin{aligned}
& V_{\mathrm{ne}}=-\hat{\imath}_{1} R_{\mathrm{ne}} \\
& V_{\mathrm{fe}}=\hat{\imath}_{8} R_{\mathrm{fe}} .
\end{aligned}
$$


As we are interested in the frequency-domain response, we need to perform this calculation for each frequency sample.

\section{B. Implementation}

In view of a simple tool, we want the user to describe the essential: the geometry of the trace and the illumination. The meshing is a repetitive task, which is a tedious and error-prone task if performed by humans. Therefore, we chose to automate it.

In order for the simulator to be easily incorporated in a PCB design tool, we preferred a scripting language that can provide an object-oriented (OO) application programming interface (API). In order to perform reproducible computational research [11], we preferred a free-to-use language. Therefore, we implemented the simulator in Python and published the code that produces the figures of this article on Github [12].

In order to mesh the transmission line, we need to decide upon the number of cells to use. For the field to be approximately uniform to each cell, we decide to take 50 cells per illumination wavelength for the highest frequency of interest. In our case study $(5 \mathrm{~cm}$ until $20 \mathrm{GHz})$, this means 167 cells. With 301 frequency points from $20 \mathrm{MHz}$ to $20 \mathrm{GHz}$, the calculation takes $24.2 \mathrm{~s}$ on an Intel $2.53 \mathrm{GHz}$ Core 2 Duo processor.

To numerically solve (8), we use the Moore-Penrose pseudoinverse implementation of NumPy, which uses singular value decomposition (SVD). About half of the total execution time is spent on this call. This and other matrix manipulations depend heavily on the matrix size.

We recognise that for low-frequencies, we do not need a great number of cells. Therefore, we decide to re-mesh the transmission line for each frequency with a certain number of cells per wavelength. For example, with 50 cells per wavelength, the simulation now only takes $1.8 \mathrm{~s}$ on the same platform.

How many cells per wavelength does one need? We used the first simulation (50 cells per wavelength, non-adaptive) as reference, and calculate the error of subsequent adaptive simulations while varying the number of cells per wavelength. Then, we calculated the log-frequency weighted average error. Finally, we calculated the log-frequency average absolute deviation from this average error. Both error measures are shown in Figure 6. In our case study, a 20 cells per wavelength resolution yields an acceptable error $(<1 \mathrm{~dB})$.

The simulation was run with 20 cells per wavelength adaptive meshing, which took $0.45 \mathrm{~s}$. The result is displayed in Figure 9.

\section{Modified TAYlor CEll}

Alternatively, we can elaborate Taylor's model analytically for the case of a grazing incident wave [3]. The result turns out to be a slightly modified Taylor cell, without the need for meshing. In the present article, the model is presented in an intuitive manner (similar to [13]); for a more rigorous underpinning of the model, please refer to [3].

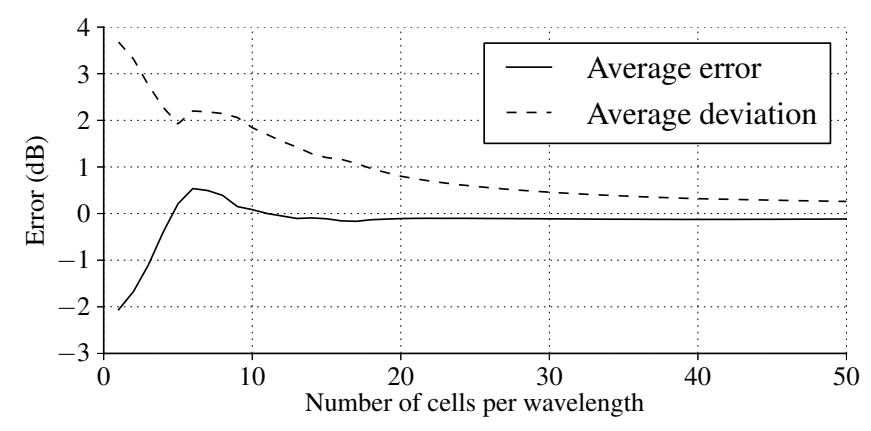

Figure 6. Sensibility of the adaptively-meshed simulation result for number of cells per wavelength.

We start with the low-frequency case, where a single Taylor cell suffices. Then we will try to imagine what happens with rising frequency. Finally, we will postulate an analytical modification on the low-frequency case to take into account high-frequency effects.

\section{A. Low-frequency Case}

Let us consider the low-frequency case using the quasi-static approximation. Because the illumination wavelength is long with respect to the line length, the field can be considered uniform along the line, and we can lump the line as a single cell $(\Delta \ell=\ell)$. Because the wavelength in the transmission line is long with respect to the line length, we can ignore the phase shift introduced by the transmission line. In our case of characteristic loads $\left(R_{\mathrm{ne}}=R_{\mathrm{fe}}=Z_{c}\right)$ we can find the eitherend terminal voltages by inspecting Figure $2 b[1]$ :

$$
V_{\mathrm{LF}}=-\frac{1}{2} \mathrm{j} \omega c E_{t} Z_{c} h \ell \mp \frac{1}{2} \mathrm{j} \omega \mu_{0} H_{n} h \ell,
$$

where $c$ is the per-unit-length (p.u.l.) capacitance of the line. Unless otherwise noted, we simultaneously present the nearend and far-end results; $\mp$ means minus for the near end and plus for the far end.

\section{B. Thought experiment}

Let us perform a thought experiment on the lossless, characteristically terminated line of Figure $2 \mathrm{a}$, illuminated from the near-end side $\left(k_{p}=+\|k\|\right)$. The illuminating field has a normalised amplitude $i$ which is just a phase lag:

$$
i(z)=\mathrm{e}^{-\mathrm{j} k_{p} z} ; \quad k_{p}=\frac{\omega}{\mathrm{c}_{0}},
$$

where $z$ is the coordinate along the line. Let us look at the far-end induced voltage, caused by a forward traveling wave on the line: the forward eigenwave. Its normalised amplitude $w$ also is a phase lag:

$$
w(z)=\mathrm{e}^{-\mathrm{j} \beta z} ; \quad \beta=\frac{\omega}{v},
$$

where $v$ is the phase speed of a wave on the transmission line.

We start at an illumination frequency where the error of a single Taylor cell is negligible, and let the frequency increase little by little. Using Figure 7, we try to imagine what happens. 

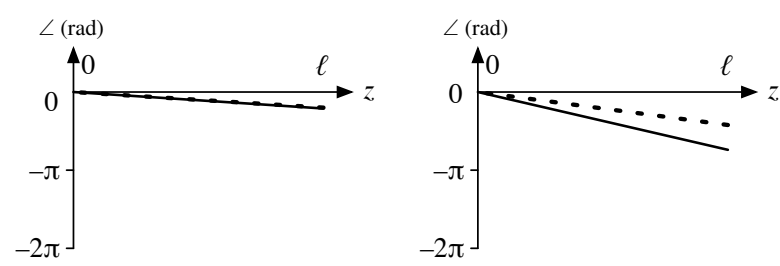

(a) Low frequency...

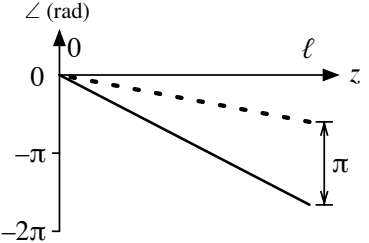

(c) $\ldots$ (b) $\ldots$

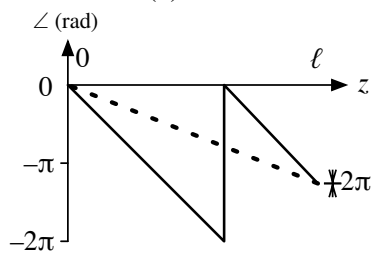

(d) ... high frequency.

Figure 7. Phase along the transmission line of the line's eigenwave $\angle w$ (solid line) and illuminating plane wave $\angle i$ (dashed line), for increasing frequency.

For low frequencies (Figure 7a), the incident field remains the same along the line, so modelling the line as one Taylor cell is legitimate.

As the frequency increases, the wavelength decreases (Figure $7 b$ ). When the wavelength is in the order of the line length, we see a propagating wave, both in free space and in the transmission line. Yet, this does not immediately invalidate the model. Indeed, the field is no longer uniform along the line, but the forward eigenwave of the line and the free space plane wave travel in the same direction. That means that, for every line slice, the free space wave and the eigenwave have approximately the same phase. Therefore, it is still reasonable to model the line as one cell.

Let the frequency increase further (Figure 7c). Now the phase difference between the forward eigenwave and the incident plane wave becomes significant; in the example shown, the phase difference goes from 0 at $z=0$ to $\pi$ at $z=\ell$. On average, both waves are still cross-correlated, but less so than for low frequencies.

In an extreme case (Figure $7 \mathrm{~d}$ ), the phase difference goes all the way from 0 at $z=0$ to $2 \pi$ at $z=\ell$. On average, the two waves are no longer cross-correlated and we expect no coupling.

\section{Modification}

So, the low frequency coupling (as predicted by a single Taylor cell) must be corrected by a measure for the lengthaverage cross-correlation between the line's eigenwave and the incident wave. This measure should be unity for low frequencies, as not to modify the low frequency coupling. This measure should amount to zero when the phase difference along the line goes all the way from 0 to $2 \pi$. Let us call this unitless measure $K$.

The cross-correlation of the incident field and the line's eigenwave amplitudes is given by the complex conjugated

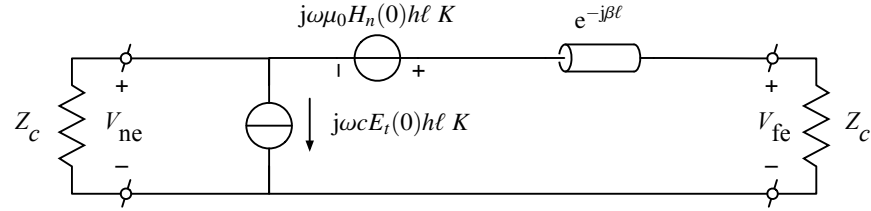

Figure 8. Modified Taylor cell, taking into account long-line effects. Note that $K$ must be selected to predict the coupling to either the forward- or the backward travelling eigenwave.

product $i w^{*} . K$ is then found by averaging along the line [3]:

$$
K=\frac{1}{\ell} \int_{0}^{\ell} i(z) \cdot w^{*}(z) \mathrm{d} z=\frac{1}{\mathrm{j}\left(k_{p} \mp \beta\right) \ell}\left(1-\mathrm{e}^{\mathrm{j}\left(k_{p} \mp \beta\right) \ell}\right) .
$$

To calculate the near-end induced voltage, the backward travelling eigenwave $w=\mathrm{e}^{+\mathrm{j} \beta z}$ was used. The resulting, modified Taylor cell is depicted in Figure 8.

This closed-form analytical solution was evaluated using a Python script [12] in $0.7 \mathrm{~ms}$.

\section{RESULTS}

The numerical results of both simulations are compared in Figure 9 . The meshed results differ by $-0.4 \mathrm{~dB}$ on average from the modified Taylor cell, with an average absolute deviation of $1.0 \mathrm{~dB}$ from this error. The two can be made to approach slightly, by more cells in meshed simulation, at the expense of greater execution time (cf. Table I).
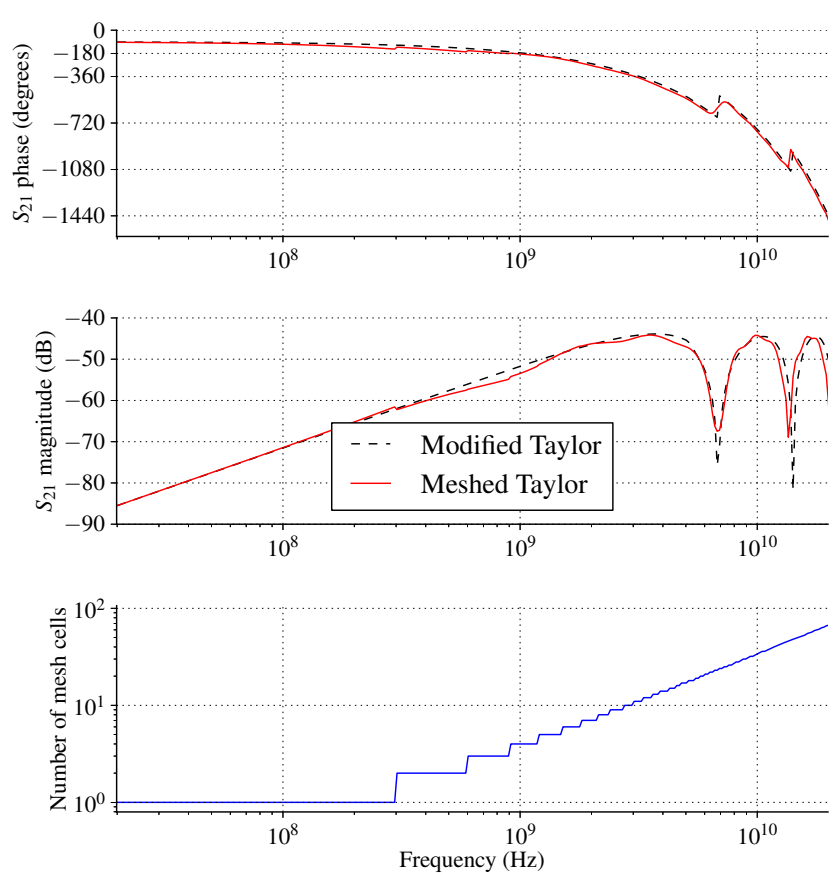

Figure 9. Simulation of the coupling from a GTEM cell input to the far end of a $5 \mathrm{~cm}$ microstrip trace. On the one hand the modified Taylor cell: the analytical solution of Figure 8 and (14). On the other hand a meshed Taylor cell, solved using Kron: the transmission line was meshed adaptively in 20 cells per free-space-wavelength (the 50- and 100-cell curves are indistinguishable). 
Table I

Correlation of Meshed and Modified Taylor Cell Simulations

\begin{tabular}{|l|l||l|l|l|}
\hline $\begin{array}{l}\text { \#Cells/ } \\
\text { wave- } \\
\text { length }\end{array}$ & Adaptive & $\begin{array}{l}\text { Meshed } \\
\text { Execution } \\
\text { Time }\end{array}$ & $\begin{array}{l}\text { Average } \\
\text { Meshed - } \\
\text { Modified }\end{array}$ & $\begin{array}{l}\text { Deviation } \\
\text { Meshed - } \\
\text { Modified }\end{array}$ \\
\hline \hline 20 & Yes & $0.5 \mathrm{~s}$ & $-0.4 \mathrm{~dB}$ & $1.0 \mathrm{~dB}$ \\
\hline 50 & Yes & $1.8 \mathrm{~s}$ & $-0.4 \mathrm{~dB}$ & $0.7 \mathrm{~dB}$ \\
\hline 100 & Yes & $9.9 \mathrm{~s}$ & $-0.3 \mathrm{~dB}$ & $0.6 \mathrm{~dB}$ \\
\hline 100 & No & $91.1 \mathrm{~s}$ & $-0.3 \mathrm{~dB}$ & $0.7 \mathrm{~dB}$ \\
\hline
\end{tabular}

\section{CONCLUSIONS AND RECOMMENDATIONS}

This paper presented two simulations of the coupling of a grazing-incident, vertically polarized plane wave to a characteristically terminated microstrip PCB trace. Both simulations are based on Taylor's model, which uses distributed voltage and current sources along the line. The first simulation automatically meshes the line in 20 cells per wavelength and solves the resulting circuit using Kron's formalism. The second simulation uses one modified Taylor cell, that does not need to be meshed to predict long-line effects.

The first simulation executes in $0.5 \mathrm{~s}$ on an $2.53 \mathrm{GHz}$ Intel Core 2 Duo processor. Potentially, real world nonidealities, like non-characteristic, frequency-dependent termination impedances and excess capacitances along the line, could easily be added to the circuit. The second method executes in $0.7 \mathrm{~ms}$ on the same platform. The difference between the first and the second method amounts $-0.4 \mathrm{~dB}$ on average, which can be slightly improved by increasing the number of cells.

To sum up: both simulations yield the same results, while a meshed Taylor cell is more flexible and a modified Taylor's cell is faster.

Future work on both methods seems interesting. As for the meshed Taylor cell: originally, we would have liked to use Branin's cells to represent the transmission line. However, it seemed that an otherwise uniform transmission line cannot be meshed with impunity into Branin's cells. Apparently, a cut in the model must correspond to some non-uniformity in the modeled line. This suggestion led Casagrande and Maurice to discover the modified Branin cell.

We here elaborated the matrices and vectors for our particular problem. Translating a circuit to a representation in Kron's formalism is a recurring and error-prone task that could be automated. One could imagine an open-source library that allows connecting circuit elements together in an object-oriented fashion. Using open libraries for symbolic calculation like sympy, symbolic simulation results may be given to the user. Apart from standard elements like resistances and capacities, there may also be circuit elements that are adaptively meshed 'under the hood' (hidden for the user).

As for the modified Taylor's cell: there is first some analytical work to be done, to take into account non-grazing incidence angles. Worst-case analysis must probably be employed to keep the solution closed-form. Moreover, it should be joined to existing microwave theory, to allow for arbitrary terminal impedances and trace discontinuities.

The speed of the closed-form calculation opens up practical possibilities. For example, the angle of incidence could be swept to produce an antenna diagram within a second. A designer could click on a PCB trace or net and almost immediately see its associated antenna diagram, instead of performing this simulation in an external full-wave solver.

By reciprocity, the far field emissions can be calculated too, if the signal levels are known by the PCB design tool.

\section{ACKNOWLEDGEMENTS}

This bit of research was made possible by the French national project SEISME (simulation of emissions and immunity of electronic systems).

\section{REFERENCES}

[1] C. R. Paul, Introduction to Electromagnetic Compatibility. Hoboken, New Jersey: Wiley, 2006.

[2] M. Leone and H. L. Singer, "On the coupling of an external electromagnetic field to a printed circuit board trace," Electromagnetic Compatibility, IEEE Transactions on, vol. 41, no. 4, pp. 418-424, 11 1999.

[3] S. T. Op 't Land, M. Ramdani, R. Perdriau, M. Leone, and M. Drissi, "Simple, Taylor-based worst-case model for field-to-line coupling," JPIER, vol. 140, pp. 297-311, jun 2013.

[4] C. A. Nucci, F. Rachidi, and M. Rubinstein, "An overview of field-totransmission line interaction," Applied Computational Electromagnetics Society Newsletter, vol. 22, no. 1, pp. 9-27, 2007.

[5] C. D. Taylor, R. Satterwhite, and C. W. Harrison, Jr., "The response of a terminated two-wire transmission line excited by a nonuniform electromagnetic field," Antennas and Propagation, IEEE Transactions on, vol. 13, no. 6, pp. $987-989$, nov 1965.

[6] A. K. Agrawal, H. J. Price, and S. H. Gurbaxani, "Transient response of multiconductor transmission lines excited by a nonuniform electromagnetic field," Electromagnetic Compatibility, IEEE Transactions on, vol. EMC-22, no. 2, pp. 119-129, 51980.

[7] F. Rachidi, "Formulation of the field-to-transmission line coupling equations in terms of magnetic excitation field," Electromagnetic Compatibility, IEEE Transactions on, vol. 35, no. 3, pp. 404 -407, aug 1993.

[8] G. Kron, Tensor Analysis of Networks. John Wiley and Sons, Inc., 1939.

[9] O. Maurice, "Theoretical application of the tensorial analysis of network for EMC at the system level," PSA Peugeot-Citroen, Tech. Rep., 2007. [Online]. Available: http://hal.archives-ouvertes.fr/hal-00166215

[10] _ _ "xTan par la pratique." [Online]. Available: http://olivier.maurice pagesperso-orange.fr/topologieAppliquee/ExTAN_for_Students_bat.pdf

[11] V. Stodden, C. Hurlin, and C. Perignon, "Runmycode.org: A novel dissemination and collaboration platform for executing published computational results," in E-Science (e-Science), 2012 IEEE 8th International Conference on, 2012, pp. 1-8.

[12] Field-to-line coupling models and measurement data. [Online] Available: https://github.com/eseo-emc/field2line

[13] S. T. Op 't Land, T. Mandić, M. Ramdani, A. Barić, R. Perdriau, and B. Nauwelaers, "Comparison of field-to-line coupling models: Coupled transmission lines model versus single-cell corrected Taylor model," in Electromagnetic Compatibility (EMC EUROPE), 2013 International Symposium on, sep 2013, pp. 276-281. 